\title{
Costing 'healthy' food baskets in Australia - a systematic review of food price and affordability monitoring tools, protocols and methods
}

\author{
Meron Lewis ${ }^{1, *}$ and Amanda Lee ${ }^{1,2}$ \\ 'School of Exercise and Nutrition Sciences, Queensland University of Technology, Victoria Park Road, Kelvin Grove, \\ QLD 4059, Australia: ${ }^{2}$ School of Public Health and Social Work, Queensland University of Technology, Brisbane, \\ QLD, Australia
}

Submitted 29 February 2016: Final revision received 26 May 2016: Accepted 29 June 2016: First published online 9 September 2016

\begin{abstract}
Objective: To undertake a systematic review to determine similarities and differences in metrics and results between recently and/or currently used tools, protocols and methods for monitoring Australian healthy food prices and affordability.

Design: Electronic databases of peer-reviewed literature and online grey literature were systematically searched using the PRISMA approach for articles and reports relating to healthy food and diet price assessment tools, protocols, methods and results that utilised retail pricing.

Setting: National, state, regional and local areas of Australia from 1995 to 2015.

Subjects: Assessment tools, protocols and methods to measure the price of 'healthy' foods and diets.

Results: The search identified fifty-nine discrete surveys of 'healthy' food pricing incorporating six major food pricing tools (those used in multiple areas and time periods) and five minor food pricing tools (those used in a single survey area or time period). Analysis demonstrated methodological differences regarding: included foods; reference households; use of availability and/or quality measures; household income sources; store sampling methods; data collection protocols; analysis methods; and results.

Conclusions: 'Healthy' food price assessment methods used in Australia lack comparability across all metrics and most do not fully align with a 'healthy' diet as recommended by the current Australian Dietary Guidelines. None have been applied nationally. Assessment of the price, price differential and affordability of healthy (recommended) and current (unhealthy) diets would provide more robust and meaningful data to inform health and fiscal policy in Australia. The INFORMAS 'optimal' approach provides a potential framework for development of these methods.
\end{abstract}

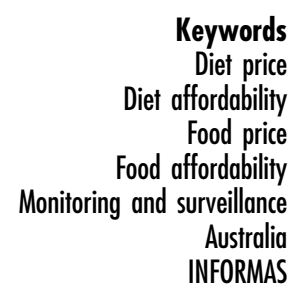

Unhealthy diet is the leading preventable risk factor contributing to the burden of disease in Australia and globally $^{(1)}$. The Australian Dietary Guidelines (ADG) ${ }^{(2)}$ provide evidence-based recommendations that aim to promote the potential benefits of healthy eating, improve community health and well-being, and reduce the risk of diet-related disease ${ }^{(2)}$. However, less than $7 \%$ of Australians consume a diet consistent with these guidelines $^{(3)}$.

A range of factors affect dietary choices, including price, availability, accessibility and promotion of foods ${ }^{(4)}$. These factors can contribute to population food insecurity, that is having inconsistent physical, social and economic access to sufficient, safe and nutritious food that meets dietary needs and food preferences for an active and healthy life ${ }^{(5)}$. Perceived low affordability has been reported as a key barrier to the purchase of 'healthy' foods, particularly in low socio-economic groups, although well-defined data in this area are lacking ${ }^{(6-8)}$. It has been suggested that food is affordable when no more than $30 \%$ of household income is required for its purchase ${ }^{(9)}$. Price elasticity studies have reported that consumers are more sensitive to price differences between close substitute foods (for example, white $v$. wholemeal bread) than to price changes between less 


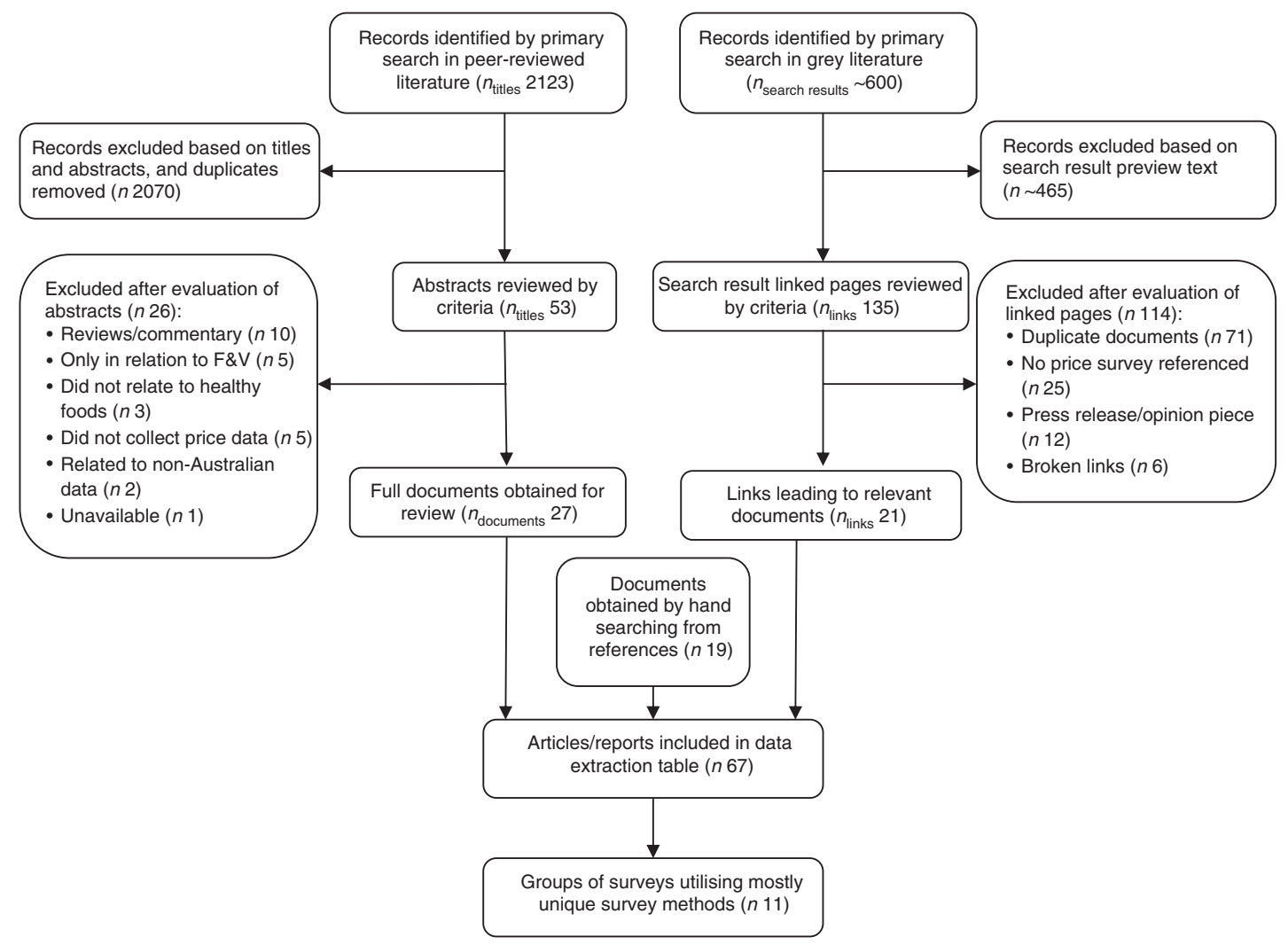

Fig. 1 PRISMA (Preferred Reporting Items for Systematic Reviews and Meta-Analyses) diagram showing the flow of studies (F\&V, fruit and vegetables)

similar foods, further suggesting that cost may play a role in the choice between healthy and unhealthy diets ${ }^{(10)}$.

Different approaches have been utilised to monitor food prices in Australia, such as Consumer Price Indexes ${ }^{(7)}$ and supermarket price surveys ${ }^{(8,11)}$ tallying and comparing the price of highly selected individual food items. A variety of 'food basket' methods that assess the cost of diets, rather than foods, have also been developed within Australia for a variety of purposes at state, regional and community levels ${ }^{(11)}$. These methods have the potential to measure the cost of a healthy diet; however, dissimilarity of metrics is a recognised barrier to the production of comparable data $^{(12)}$ that would help pinpoint areas of high food insecurity and better inform policy and practice in relation to food pricing and affordability.

A stepwise food price and affordability monitoring framework has been developed by the International Network on Food and Obesity/non-communicable disease Research, Monitoring and Action Support (INFORMAS) network ${ }^{(8)}$. The framework advocates 'minimal', 'expanded' and 'optimal' monitoring approaches, including measurement indicators of affordability, stratification by region and socio-economic status (SES), and representative sampling. This approach provides potential for robust national data benchmarks and international comparisons. To assist in the development of national food price and affordability monitoring tools, protocols and methods for use in Australia consistent with the INFORMAS framework, we conducted a systematic review to determine similarities and differences in the metrics and results between recently and/or currently used tools and protocols for monitoring Australian 'healthy' food prices and affordability.

\section{Methods}

The systematic review was conducted based on the PRISMA (Preferred Reporting Items for Systematic Reviews and Meta-Analyses) statement ${ }^{(13)}$ and included peerreviewed and grey literature published from 1995 to 2015 (Fig. 1).

Seven electronic databases were searched: Informit Health; MEDLINE, PubMed; EMBASE; CINAHL; Web of Science: Science Citation Index and Conference Proceedings Citation Index; and the Cochrane Library. Grey literature was searched using Google.com.au advanced searches, limited to the web address extensions of .gov.au, .org.au, .net.au and .com.au. The search terms used were 'health*', 'food', 'diet*', 'cost/price/afford*', 'healthy food' and 'healthy diet', with additional location limiting to Australia where necessary. Following identification of pertinent results, reference lists were reviewed and hand searching identified other known documents. Search 
results for the same journal article or web links to the same report were excluded as duplicates. However, discrete journal articles and reports that related to the same collected data set were included.

The initial search results were reviewed against the inclusion criteria of relating to 'healthy' food and diet price assessment tools, protocols, methods and results using methods of retail pricing and being pertinent to Australia (at a national, state, regional or local community level). 'Healthy food price assessment' was defined as the costing or pricing of a list, or basket, of foods that represent a 'healthy' diet for one or more persons. Thus studies relating to comparative pricing of selected individual 'healthy' and 'unhealthy' foods were not included ${ }^{(14)}$. Articles and reports relating to household food expenditure methods, interventions to alter purchasing habits and dietary improvement modelling were excluded. Additionally excluded were: reviews, commentaries, press releases and opinion pieces; surveys solely relating to fruit and vegetables (F\&V); and surveys where no price data were collected. Poster presentations were also excluded due to their providing insufficient information in relation to the survey methods.

Copies of three reports identified through hand searching $^{(15-17)}$ and reports of the Northern Territory Market Basket (NTMB) surveys for 1998-2002 and 2013 were not publicly accessible, so were excluded from the analysis.

The systematic search identified thirty-nine reports and twenty-four journal articles which described fifty-nine discrete healthy food pricing surveys undertaken within Australia. Some of these surveys were reported in the grey literature as well as in one or more published journal articles. In addition, some journal articles described a time series of surveys.

The contents of the journal articles and reports were analysed to determine all available information relating to the basket contents (the food pricing tool), representative households, source of household income, sampling of stores for the survey, timing of data collection, process instructions for data collection (e.g. brands or generic brands, missing item protocols), data analysis methods and reported results.

Due to the broad scope of the review, large volumes of data were identified from the reports and articles. The data were transcribed into separate tables according to the type of information listed above. Within each table, the survey data are ordered by the food pricing tool, then geographical reach and then time. Two summary tables are included in the body of this manuscript; the remaining data are included in the online supplementary material due to the large size of the data sets.

\section{Results}

\section{Food pricing tool}

Analysis of the fifty-nine discrete surveys (Table 1) identified six 'major' food pricing tools (defined as those used in multiple areas and at multiple time periods) and five 'minor' food pricing tools (defined as those used in only one survey area or at one time period). Surveys were conducted to assess food prices for different purposes at various geographic levels: statewide, regional or local. Each survey area included one or more local government areas, with one or more stores included within each local government area. All state/territory-wide surveys used a major food pricing tool by definition. The two food pricing tools developed most recently, the Revised Queensland Healthy Food Access Basket (HFAB) ${ }^{(18)}$ and the Healthy and Sustainable (H\&S) basket developed by Friel et al. ${ }^{(19)}$, included more contemporary methodology than the other food pricing tools.

Table 2 shows that all of the food pricing tools, with the exception of the H\&S basket ${ }^{(19)}$, measured the price of

Table 1 Summary of the major food pricing tools $(n 5)$ and minor food pricing tools $(n 6)$ used in Australia

\begin{tabular}{|c|c|c|c|}
\hline & Food pricing tool & Abbreviation & Survey areas used (number of time periods tool used in each survey area) \\
\hline \multirow[t]{6}{*}{ Major* } & $\begin{array}{l}\text { Queensland Healthy Food Access } \\
\text { Basket }^{(20)}\end{array}$ & QLD HFAB & $\begin{array}{l}\text { Statewide QLD (5); statewide NSW (3); south-west rural VIC (1); } \\
\text { Bundaburg, QLD (1); Zillmere, QLD (1); Yarra, VIC (1) }\end{array}$ \\
\hline & $\begin{array}{l}\text { Western Australia Food and Cost } \\
\text { Access Survey }\end{array}$ & WA FACS & Statewide WA (2) \\
\hline & Northern Territory Market Basket ${ }^{(24)}$ & NTMB & Territory-wide NT (11); remote SA (1) \\
\hline & Illawarra Healthy Food Basket ${ }^{(11)}$ & IHFB & Illawarra, NSW (6); Dandenong, VIC (1); Adelaide, SA (1) \\
\hline & Victorian Healthy Food Basket ${ }^{(22)}$ & VHFB & $\begin{array}{l}\text { Rural and regional VIC (1); local areas VIC (15); Adelaide, SA (1); } \\
\text { statewide TAS (1); local area TAS (1) }\end{array}$ \\
\hline & $\begin{array}{l}\text { QLD } 2014 \text { Revised Healthy Food } \\
\text { Access Basket }{ }^{(18)}\end{array}$ & $\begin{array}{l}\text { Revised QLD } \\
\text { HFAB }\end{array}$ & Statewide QLD (1) \\
\hline \multirow{5}{*}{ Minor† } & Healthy and Sustainable Basket ${ }^{(19)}$ & H\&S basket & Western Sydney, NSW (1) \\
\hline & $\begin{array}{l}\text { Sydney Food Fairness Alliance Market } \\
\text { Basket Survey }\end{array}$ & SFFA & Western Sydney, NSW (1) \\
\hline & Kettings Meal Plan ${ }^{(34)}$ & Kettings & Melbourne, VIC (1) \\
\hline & $\begin{array}{l}\text { Katoomba Food Retail Price Mapping } \\
\text { Project }^{(33)}\end{array}$ & Katoomba & Katoomba, NSW (1) \\
\hline & Food Supply in rural South Australia ${ }^{(23)}$ & Meedeniya & Rural SA (1) \\
\hline
\end{tabular}

*Major: food pricing tools used across multiple survey areas and multiple time periods.

†Minor: food pricing tools used in one survey area or at one time period, with some minor variations. 
Table 2 Contents and representative households of the food pricing tools used in Australia

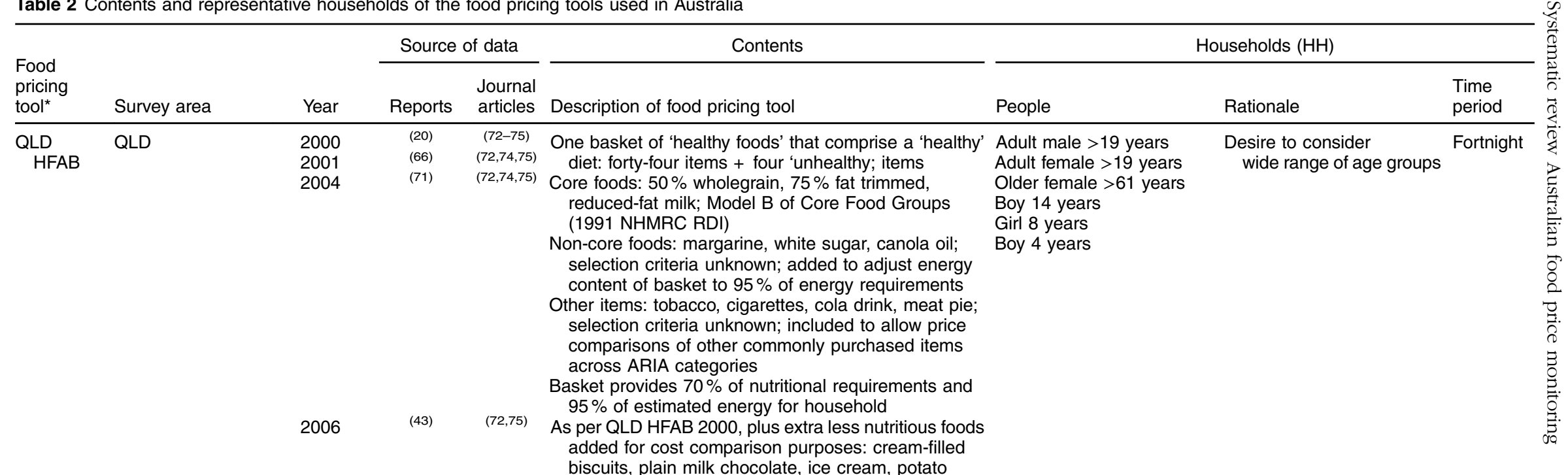

\begin{tabular}{|c|c|c|c|c|}
\hline & 2010 & (44) & (72) & $\begin{array}{l}\text { As per QLD HFAB 2006, excluding ice cream (unclear } \\
\text { why ice cream excluded) }\end{array}$ \\
\hline NSW & 2006 & (35) & $\begin{array}{l}(36) \\
(36)\end{array}$ & As per QLD HFAB 2006 \\
\hline South-west VIC & $\begin{array}{l}2009 \\
2002\end{array}$ & & $\begin{array}{l}\text { (36) } \\
\text { (48) }\end{array}$ & As per QLD HFAB 2000, plus ten foods from list of top \\
\hline & & & & $\begin{array}{l}\text { items purchased in supermarkets in Australia in } 2000 \\
\text { ('unhealthy' foods for comparison to QLD HFAB and } \\
\text { indicator of access to food in rural communities) }\end{array}$ \\
\hline Illmer & 2008 & (39) & & As per QLD HFAB 2000 \\
\hline Bundaberg, QLD & 2008 & (40) & & As per QLD HFAB 2000 (tobacco, cigarettes and meat \\
\hline
\end{tabular}

Yarra, VIC (no date)

(31) As per QLD HFAB 2000, some items replaced with common ethnically specific/appropriate foods with nutrient equivalence (rationale for inclusion not reported)

List of 190 different foods, prices of 430 items collected, with at least three brands collected for each food (increases likelihood of at least one price collected per store per type of food and common practice in ABS pricing survey methodology). Includes all items of QLD HFAB, NTMB and IHFB, to allow selection of
'healthy' baskets during analysis for comparison with previous surveys

Foods include: commonly purchased F\&V (criteria not reported); foods preferred by Indigenous

communities (criteria not reported); top five breakfast cereals \& top four lunch box snacks (selected from Choice reviews, 2009, 2010); top selling market

\author{
$\mathrm{HH} 1: 2 \times 40$-year-olds $+2 \quad$ Not reported \\ $\times$ kids aged $12 \& 7$ years \\ $\times$ kids aged $12 \&$ \\ 7 years \\ Week
}




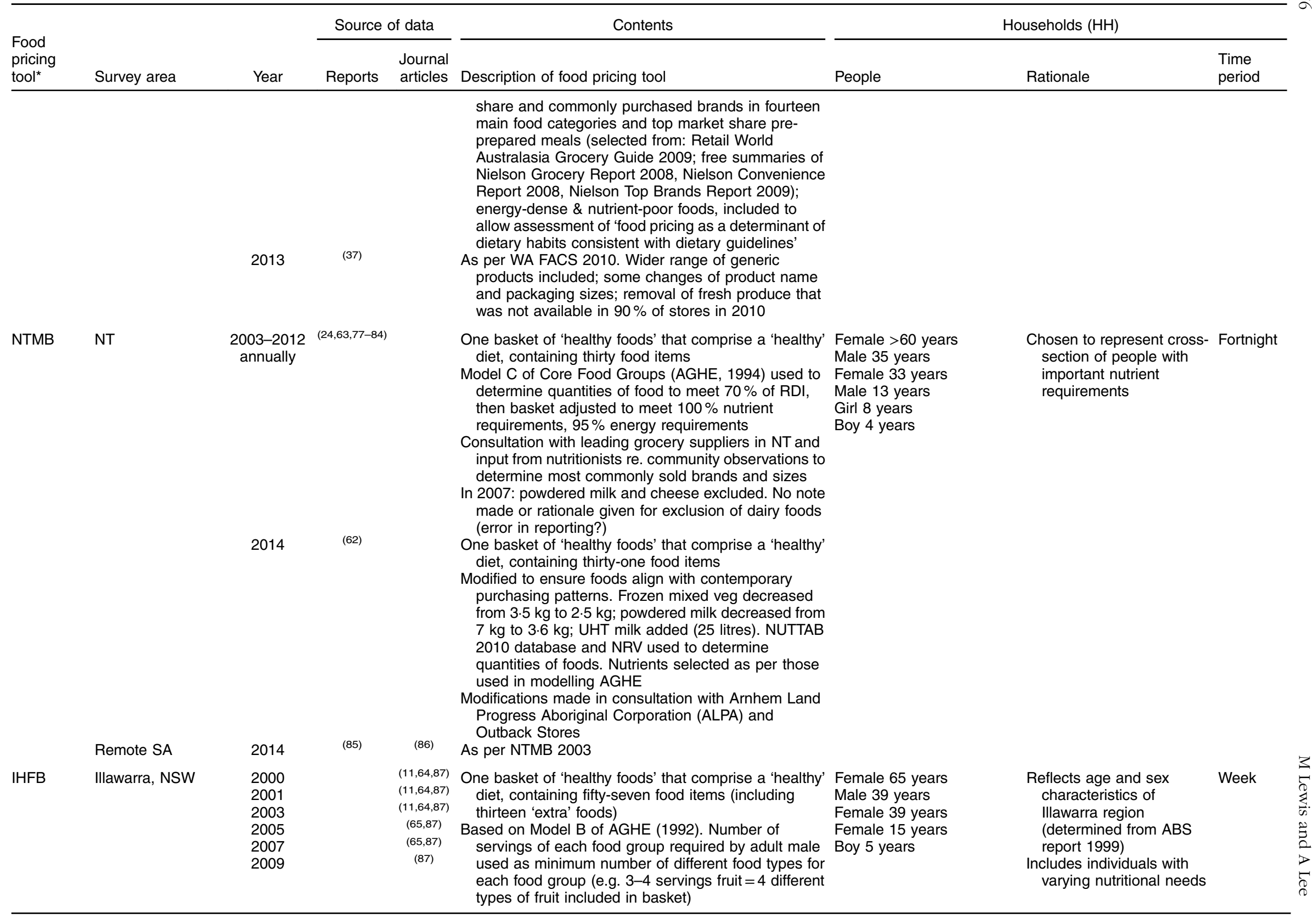




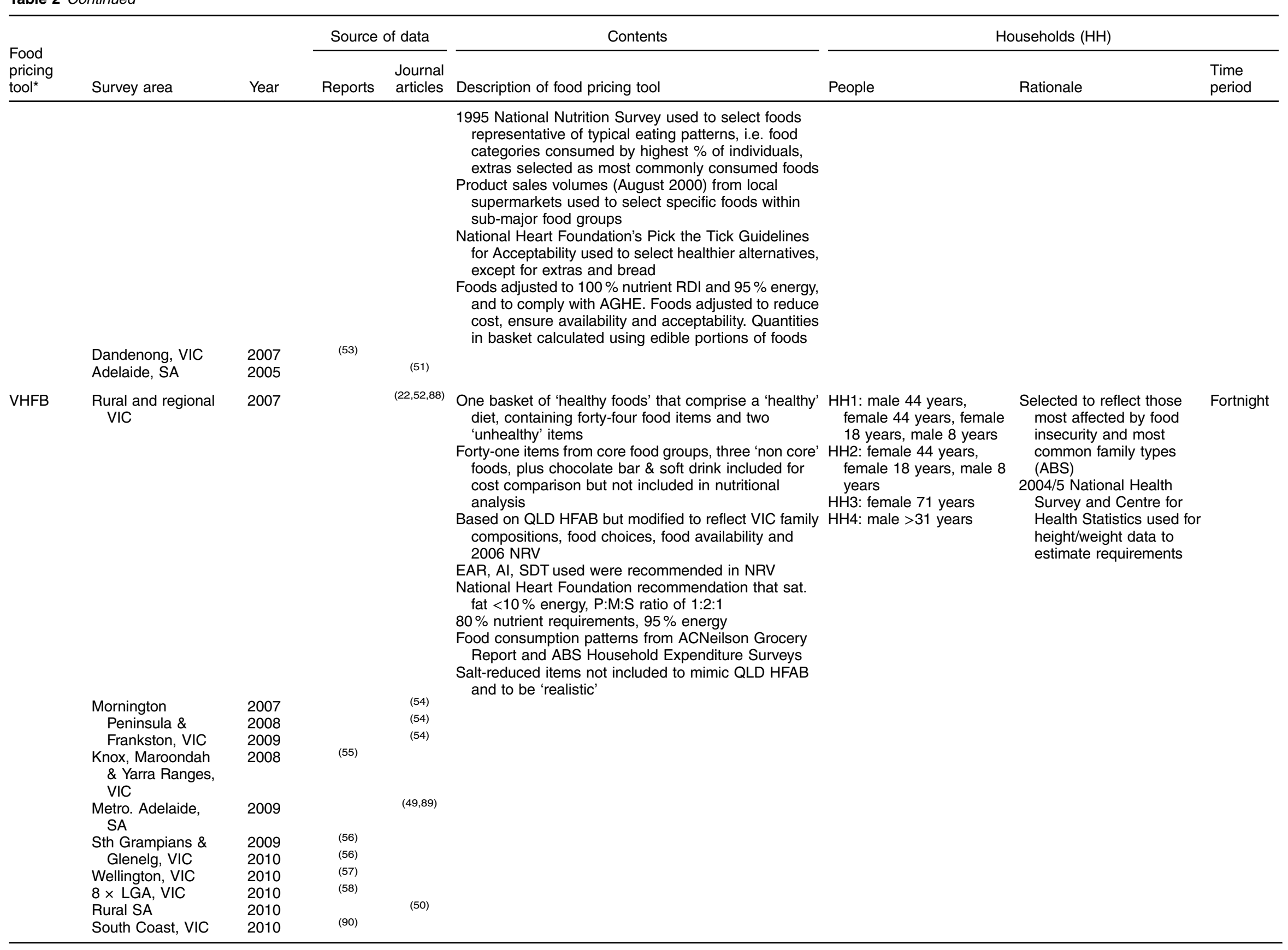




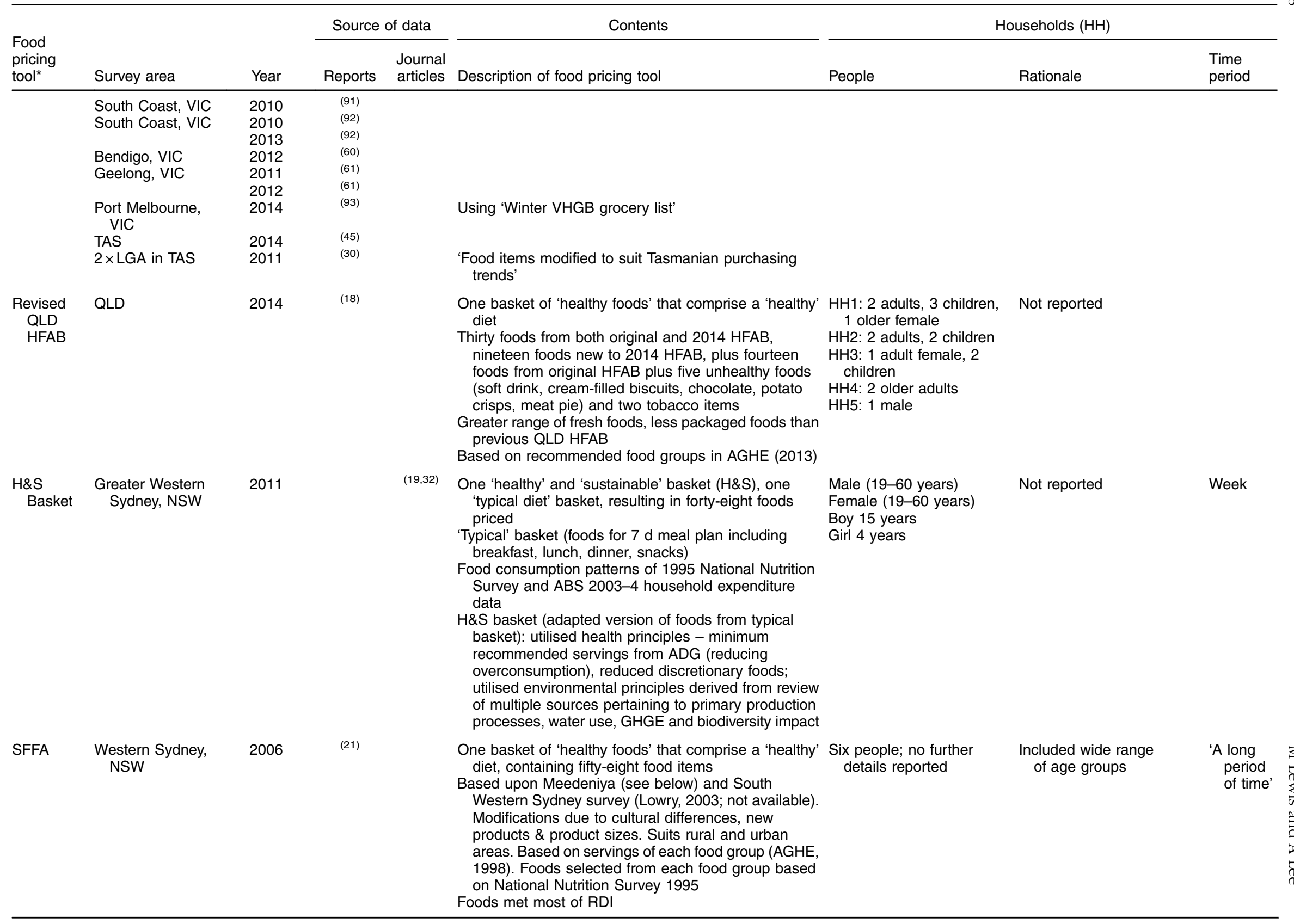




\section{Katoomba Katoomba, NSW (no date)} Project

\section{Meedeniya Rural SA}

(23)
Contents

Journal

articles Description of food pricing tool

'Healthy' $7 \mathrm{~d}$ meal plan developed; all foods of mea plan priced and actual meal/snack calculated

To calculate cost of meal plan based on AGHE, NRV, recipes used from consumer-targeted cooking and budgeting resources (Nutrition Australia)

Meal plan supplies breakfast, lunch, dinner, snacks \& extras with $100 \%$ nutrient requirements and $95 \%$ energy requirements

Model B of AGHE

Excludes alcohol and takeaway foods

List of $\sim 200$ foods for price collection, three baskets developed (one for each type of household)

Basket for $\mathrm{HH} 1$ : thirty-seven items + fifteen miscellaneous items (cost considered over 1 month) Hears, child 4 years

Basket for $\mathrm{HH} 2$ : thirty-three items + fifteen 7 years, child 4 years miscellaneous items (cost considered over 1 month) HH3: male 70 years Basket for HH3: twenty-five items + thirteen miscellaneous items (cost considered over 1 month) Basket contents selected to meet needs as per AGHE (no further details reported)

Foods included based on being 'best value' for that particular food within food group

'Healthier' options included where available (low salt, low fat, high fibre)

One basket of 'healthy foods' that comprise a 'healthy' diet, containing fifty-one food items and six snack foods (biscuits, crisps and soft drinks, cake, chocolate, ice cream)

Basket items based on the AGHE food groups

Foods within each food group were based on the foods actually eaten as per National Nutrition Survey 1995 Meet RDI for most nutrients

People

female 40 years, female

12 years, male 7 years

female 12 years, male

7 years

11: male 30 years,
Two adults

Boy 14 years

Girl 8 years

Boy 4 years

Woman $>54$ years
Households $(\mathrm{HH})$

Rationale

period

Based on ABS 2003 Family Week

Characteristics Survey

and 2006 Population by

Age and Sex.

Anthropometric dat

from ABS National

Health Survey and NRV

Not reported

Week

Council; RDI, Recommended Dietary Intake; ARIA, Accessibility/Remoteness Index of Australia; ABS, Australian Bureau of Statistics; F\&V, fruit LGA, local government area; NHMRC, National Health and Medical Research Councl, RD, Retrient Reference Value; EAR, Estimated Average Requirement; Al, Adequate Intake; SDT, suggested dietary target; P:M:S, polyunsaturated:monounsaturated:saturated fat; ADG, Australian Dietary Guidelines; GHGE, greenhouse gas emissions.

*See Table 1 for explanation of food pricing tool abbreviations. 
a 'healthy' basket of food items developed to reflect a 'healthy' diet, which was informed by the principles of the national dietary guidelines ${ }^{(11,20-24)}$. Model $\mathrm{B}$ of the Core Food Groups ${ }^{(25)}$ of the $1992 \mathrm{ADG}^{(26)}$ was used by all these tools except the Revised QLD $\operatorname{HFAB}^{(18)}$, which adjusted the basket contents to meet new modelling ${ }^{(27)}$ developed to inform the revision of the ADG in $2013^{(2)}$.

The Western Australia Food and Cost Survey (WA FACS) food pricing tool ${ }^{(28)}$ conceptually falls within the group that measured a single 'healthy' basket, although the tool included a very comprehensive list of foods for pricing that produced a database encompassing the contents of similar 'healthy' baskets, such as the QLD $\mathrm{HFAB}$, to potentially enable comparison with the results of surveys using these food pricing tools.

All tools, except the H\&S basket and the Revised QLD HFAB, described 'healthy' baskets that included several 'unhealthy' non-core or discretionary foods (energydense, nutrient-poor foods not required for health that are high in added sugar, saturated fats, salt and/or alcohol ${ }^{(2)}$ ), such as sugar and oil ${ }^{(20,24)}$, to adjust the energy content of the basket, and also included commonly consumed discretionary foods, such as sausages ${ }^{(21)}$, cake ${ }^{(11,23)}$ or chocolate $^{(23)}$. Thus most 'healthy' baskets do not constitute a diet consistent with current ADG recommendations. This was acknowledged by authors of one of the Australian food pricing tools ${ }^{(20)}$, but not others.

In addition, five of the major ${ }^{(11,18,20,22,28)}$ and one of the minor ${ }^{(23)}$ food pricing tools included a separate, arbitrary group of 'unhealthy' foods, such as soft drinks, meat pies or chocolate, to try to compare pricing with the 'healthy' basket. These items were highly selective and few in number, and were not reported as intending to constitute an 'unhealthy diet'.

In contrast to these single basket approaches, the H\&S basket ${ }^{(19)}$ developed both a 'typical' basket and a 'healthy and sustainable' basket. The contents of the 'typical' basket was informed by dietary patterns observed from the 1995 Australian National Nutrition Survey ${ }^{(29)}$. To create the 'healthy and sustainable' basket, many of the 'typical' basket items were substituted with foods aligning both with the ADG (2013) and selected environmental sustainability principles, such as consideration of environmental impacts of production processes (carbon footprint, water usage, biological diversity). Therefore the H\&S study did not include tinned and frozen foods and excluded other foods considered healthy in the ADG, such as sweet potato, cauliflower and capsicum, due to their environmental impact compared with other vegetables.

Different research groups often adapted food pricing tools for use outside the original geographical locations for which they were developed without reporting detailed rationale. For example: the Victorian Healthy Food Basket (VHFB) is based upon the QLD HFAB, with unspecified contents varied to include 'local food choices' and to 'ensure availability' ${ }^{\text {(22); }}$ subsequent application of the
VHFB tool in Tasmania included unspecified 'local food choices $^{\text {(30) }}$; and contents of the QLD HFAB were adjusted for unspecified local cultural differences for use in inner-city Melbourne, Victoria ${ }^{(31)}$.

\section{Housebold composition}

Identification of a reference household for the survey area population is required in order to inform the quantity of foods in the basket to be costed. Six different household structures were described across the eleven food pricing tools, ranging from a household of two adults, three children and an older female, to a household of a single male. Three of the major ${ }^{(11,20,24)}$ and three of the minor ${ }^{(21,23,32)}$ food pricing tools included one household, whereas the other food pricing tools included between two and five different households.

The ages and gender of the adults and children within the reference households varied, which has implications for household nutritional requirements and food quantities. However, gender ${ }^{(21,23,28,33)}$ and ages ${ }^{(21,23)}$ of all reference household members were not consistently reported. A common rationale for selection of reference household composition was inclusion of a variety of age groups $^{(20,21,23)}$ and a cross-section of people with specific nutrient requirements ${ }^{(24)}$. Some food pricing tools selected reference households that were considered to reflect the usual characteristic families of the survey area ${ }^{(11,22,34)}$. No rationale was reported in relation to household composition for three of the food pricing tools ${ }^{(28,32,33)}$.

\section{Availability}

All of the food pricing tools assessed availability of all basket items. Four of the major ${ }^{(18,20,24,30)}$ and one of the minor $^{(23)}$ food pricing tools assessed availability of specific F\&V. Two major food pricing tools ${ }^{(20,28)}$ also measured the availability of food items considered a 'better nutritional choice' than common items, such as wholemeal bread or reduced fat milk. (See online supplementary material, Supplemental Table 1.)

Authors suggested that availability of healthy foods should be reported, as poor availability can indicate food insecurity ${ }^{(20,21,24,28)}$. Similarly, measurement of availability of 'better nutritional choices' in remote and very remote areas was considered desirable ${ }^{(20)}$.

\section{Quality assessment}

Subjective assessment of $\mathrm{F} \& \mathrm{~V}$ quality in stores was undertaken in four major ${ }^{(24,28,30,35,36)}$ and two minor ${ }^{(21,23)}$ food pricing surveys. Only the WA FACS tool attempted to assess meat quality ${ }^{(28,37)}$.

\section{Housebold income}

In order to determine the level of affordability of the 'healthy' baskets, most surveys, at least once, included 
estimation of reference household income. (See online supplementary material, Supplemental Table 2.) Indicative household income was calculated from Australian welfare data $^{(38)}$ in four food pricing surveys ${ }^{(18,23,24,33)}$. Three others included estimation of paid employment income $e^{(19,39,40)}$ and both methods were applied with three major $^{(11,22,28)}$ and one minor ${ }^{(34)}$ food pricing tools.

Calculation of the welfare income appeared to be based on the assumption that none of the adults in the households were employed. While limited information was reported, it appears that only the main fortnightly welfare payments, such as unemployment benefits and the aged pension, were usually included from published sources $^{(41,42)}$. In contrast, the Revised QLD HFAB food pricing tool included all possible annual payments in addition to the main payments ${ }^{(18)}$.

\section{Store selection}

Store sample selection tended to be influenced by the survey rationale. Statewide or territory-wide surveys aimed to investigate the effect of locality and remoteness on food price and availability, regional surveys looked more at the effect on price by SES within the survey area, and local area surveys often focused on food security. Hence, the methods used for selecting participating stores varied widely, and were independent of the food pricing tool used. (See online supplementary material, Supplemental Table 3.) Further, different store sampling techniques were used in different time periods for otherwise similar surveys; for example, the QLD HFAB statewide surveys changed from convenience sampling $(2000,2001,2004)$ to representative sampling in 2006 and $2010^{(43)}$.

No nationally representative food price surveys have been conducted across the whole of Australia. Only statewide surveys of Queensland ${ }^{(18,43,44)}$, Western Australia $^{(28,37)}$ and Tasmania ${ }^{(45)}$ utilised representative sampling techniques. In these studies towns or stores were stratified according to the Socio-Economic Indexes for Areas (SEIFA) ${ }^{(45)}$ or Accessibility/Remoteness Index of Australia (ARIA) classifications ${ }^{(18,28,37,43,44)}$, then locations were randomly selected within these stratifications. SEIFA ranks statistical areas of Australia by a range of socio-economic variables including household income, education, employment, occupation and housing ${ }^{(46)}$. ARIA provides a geographical measure of remoteness based upon the access to a service centre of a defined population size ${ }^{(47)}$. The Queensland and Western Australia surveys determined sample sizes to detect differences of $10 \%$ between remoteness categories at $P<0.05$ and $90 \%$ power, and oversampled very remote localities. The Tasmania survey oversampled stores in the lowest SEIFA tertile.

Other statewide surveys (in New South Wales, Northern Territory and early Queensland studies) used convenience sampling; these also selected the largest store in each included town, or district ${ }^{(20,24,48)}$, within the survey area. All of the regional surveys used convenience sampling, with occasionally some grouping based on SEIFA classifications, prior to including all stores ${ }^{(32)}$, a store from each major supermarket chain ${ }^{(21,49,50)}$ or the largest store ${ }^{(11,51)}$ within those localities.

One regional survey in south-western Victoria $^{(48)}$ included all food outlets in towns with a population of more than 100 residents. One statewide survey in New South Wales simply selected supermarkets based on the preferences of volunteer data collectors ${ }^{(36)}$ and one regional survey of rural and regional Victoria utilised convenience sampling based on the placement locations of student data collectors ${ }^{(52)}$.

Local area sampling methods mainly included every supermarket within the bounds of the survey area ${ }^{(30,31,33,39,40,53-59)}$. Two local methods included one supermarket from both of two major chain stores from each locality within the local area ${ }^{(21,34)}$. Two reports of local area surveys did not provide any information regarding the criteria for store selection ${ }^{(60,61)}$.

Data collection periods occurred in all months except January. In surveys across multiple localities, data were collected within a maximum three-month window. To reduce potential effects of seasonality, time series data were usually collected in the same months. However, one survey (NTMB) deliberately altered the timing of the data collection period in one year to try to test for seasonality effects $^{(62)}$.

\section{Data collection protocols}

Brands

Studies noted that the use of specified common brands helped ensure both consistency of data collection in all stores on all occasions and comparability over different time periods. The most common protocols (four of the major ${ }^{(11,18,20,22)}$ and all minor food pricing tools) specified recording the price of the cheapest brand (not generic) for each food item. Generic brand prices were collected separately, or included as the cheapest brand in four major $^{(11,18,20,28)}$ and three minor ${ }^{(19,33,34)}$ methods. The WA FACS protocols ${ }^{(28)}$ included collection of multiple brand name prices and a generic brand price for each food item, to increase the likelihood of collecting at least one price in each store. The collection of sale prices was excluded from all but two major ${ }^{(28,45)}$ and one minor ${ }^{(33)}$ food pricing tool. (See online supplementary material, Supplementary Table 4.)

\section{Size}

Product size protocols were reported for all major and three minor ${ }^{(21,23,34)}$ food pricing tools; a 'standard or medium size' was reported for one of these minor tools ${ }^{(34)}$. Product size protocols were not reported for the remaining two minor tools ${ }^{(19,33)}$. 
If the specified product size was not available, data collectors were instructed to select the next smallest size for four of the major ${ }^{(11,18,20,22)}$ and one minor ${ }^{(23)}$ food pricing tools. Instructions to choose either a smaller or larger size were reported for two major ${ }^{(24,28)}$ and one minor $^{(21)}$ food pricing tools. Missing item protocols were not reported for the remaining three minor food pricing tools ${ }^{(19,33,34)}$.

\section{Data analysis protocols}

To derive the cost of the 'healthy' basket, each food pricing survey determined the unit price of each food item and multiplied the unit price by the quantity required for each reference household. (See online supplementary material, Supplemental Table 5.) Analysis of the cost of 'healthy' meals was not reported in any of the food pricing surveys reviewed.

If an item was not available at a store, an average price of that item in similar locations was calculated. Similar locations were defined as stores: in the same ARIA category ${ }^{(20)}$; in the same local area ${ }^{(57)}$; of the same type ${ }^{(40)}$; in the total sample. Details of analysis protocols for missing items were not reported for most surveys ${ }^{(11,18,28,33,34)}$.

\section{Reporting of results of food pricing surveys}

As presented above, the food pricing surveys undertaken around Australia utilised a wide variety of tools, protocols and methods; consequently, a range of non-comparable findings have been reported. Reported results included: entire basket price; cost of food groups within the basket; cost of extras or 'less healthy' foods; availability of basket items; availability and variety of F\&V and/or better nutritional choices; and F\&V quality scores. Those surveys that described household income also reported on affordability of the total basket and food groups. (See online supplementary material, Supplemental Table 6.)

\section{Cross-sectional results}

Depending on the purpose of each survey and its geographic reach, results were stratified by ARIA and/or SEIFA/SES classifications, or reported for districts, local government areas, census collection districts or suburbs. In general, studies stratified by ARIA reported 'healthy' diet basket prices between 20 and $60 \%$ higher in very remote areas compared with major cities. However, no association between location and basket price was found where studies stratified results by SEIFA/SES classification. On the other hand, the study using the H\&S diet basket ${ }^{(32)}$ reported that the cost difference between the 'healthy' and the 'typical' basket was greater in disadvantaged areas.

Generally it was reported that between 25 and $40 \%$ of the income of welfare-dependent family households was required to purchase the diet baskets, with a lower amount, about $18 \%$ of income, required for single older person households.
Where assessed, it was reported generally that availability and quality of F\&V and availability of 'better nutritional choices' were lower in remoter locations, and that more items were missing in small supermarkets and convenience stores than major supermarkets.

\section{Time series results}

Reports of temporal changes were possible in surveys utilising the same methods in the same area over time. While annual price differences occasionally decreased (usually due to recovery of prices of fresh produce following natural disasters ${ }^{(63)}$ ), as would be expected long-term trends showed increasing diet basket prices.

Affordability of the 'healthy' diet basket was reported over four years in Western Australia ${ }^{(28)}$; over seventeen years in Northern Territory ${ }^{(24)}$; over ten years in Illawarra, New South Wales ${ }^{(11)}$; and over three years in a local area in Victoria ${ }^{(54)}$. No significant trends in affordability were reported in the Northern Territory and Illawarra surveys; however, the shorter Western Australia and local Victoria surveys found affordability decreased slightly but significantly over time for welfare-dependent families.

Only in the Northern Territory ${ }^{(62)}$ was improved availability and quality of $F \& V$ reported over time.

\section{Comparison between survey areas}

Few comparisons of results across different geographical areas have been reported, even where technically possible in the WA FACS survey ${ }^{(28)}$. Surveys using the Illawarra Healthy Food Basket (IHFB) tool in the Illawarra region ${ }^{(64,65)}$ compared results with Queensland ${ }^{(43,66)}$; however, only proportional changes over a similar time period could be reported due to methodological differences.

\section{Discussion}

The present review identified that six major and five minor food pricing tools and a wide range of protocols have been applied on a statewide, regional and local area basis in fifty-nine surveys in Australia. The reviewed surveys measured the cost of a basket of 'healthy' foods representing 'healthy' diets and have been able to provide answers to questions relating to relative price in different locations (more expensive in rural and remote areas than in capital and regional cities), relative price in disadvantaged areas (not significantly different), relative price over time (food prices increased) and affordability over time (relatively consistent). However, without comparison with the cost and affordability of currently consumed ('unhealthy') diets ${ }^{(7)}$, it is challenging for these data to help inform potential policy approaches ${ }^{(8)}$. Measurement of the cost of a typical 'unhealthy' diet was not undertaken by any of the reviewed surveys except the H\&S basket ${ }^{(32)}$. The price and availability of some better nutritional 
choices' or 'unhealthy' food items were included in some food pricing tools, but these measurements are insufficient to represent a 'current' or 'unhealthy' diet for comparison purposes $^{(8)}$

Governments have the potential to manipulate food prices to encourage consumption of 'healthy' foods via policies such as: taxation systems (tax liabilities on 'unhealthy' foods or tax exemptions of 'healthy' foods); agricultural or transport subsidies; or direct subsidies to high-risk populations such as provision of vouchers for healthy foods ${ }^{(8)}$. However, robust and relevant data are needed to inform policy action in relation to food pricing and taxation to improve the relative cost of healthy foods. Core to the INFORMAS stepwise food price and affordability monitoring framework ${ }^{(8)}$, at all levels, is measurement of the size and direction of the price differential between 'healthy' and 'less healthy' foods, meals or diets. Optimally, this approach utilises 'healthy' diets derived according to national dietary guidelines and 'less healthy' diets derived from national dietary intake data ${ }^{(8)}$. Use of this approach across Australia to provide a relationship between the price differential and SEIFA or ARIA stratification has the potential to provide the necessary data to inform policy.

While there were some similarities between the tools and protocols applied in the surveys, there were also many differences, even when the same food pricing tool was used in different survey areas or at a different time period. Methodological differences were found in: selection of 'healthy' basket contents; reference household composition; inclusion of availability and/or quality measures; household income sources; store sampling methods; season of data collection; and data collection protocols and analysis. As has been identified previously, it is not possible to compare results across different surveys, even when the same food pricing tool has been applied ${ }^{(9,12,67)}$.

Lack of comparability of survey results is often due to alterations in a chosen food pricing tool to accommodate local population differences. The rationale for these alterations and selection of replacement foods were not clearly reported and appeared quite subjective. Additionally, the items within the food pricing tools were selected according to various criteria to represent a 'healthy' diet and it is difficult to see how alterations for local preferences will provide additional policy-relevant data.

The review found that ten of the eleven identified food pricing tools do not fully align with the principles of the $2013 \mathrm{ADG}^{(2)}$, as discretionary foods and/or commonly consumed unhealthy foods were included within the defined 'healthy' basket. The H\&S basket food pricing tool ${ }^{(32)}$ does align with the most recently revised $\mathrm{ADG}^{(2)}$ but specifically incorporates more exacting environmental sustainability principles, which appear to increase diet costs.

All of the food pricing tools, with the exception of the Revised QLD HFAB and the H\&S basket, are based upon outdated dietary guidelines. The Revised QLD HFAB tool is also consistent with the healthy diet tool proposed under the optimal approach of the INFORMAS food price and affordability monitoring framework ${ }^{(8)}$.

In contrast to the measurement of basket item availability, the availability of F\&V and 'better nutritional choices', and food quality, were not consistently measured by the surveys. Other studies looking at F\&V quality and availability alone were identified during the literature search; however, these have not been included as they did not represent a complete diet. A separate review of these studies may be required.

Affordability of healthy foods has been assessed inconsistently. Determination of affordability requires calculation of household income, which will vary according to composition of the household and assumptions made regarding the working status and income source of household members. The use of government welfare payments, minimum wage levels or median household income to estimate household income also requires application of arbitrary assumptions. Therefore, comparisons will be challenging until methods are standardised. However, given the well-established social gradient of health associated with dietary quality, measuring the affordability of 'healthy' diets in relation to household income would provide a useful benchmark ${ }^{(8)}$. When assessed, affordability of the 'healthy' baskets was just below or over the suggested $30 \%$ of household income level ${ }^{(9)}$. For comparison, it would also be useful to assess affordability of actual dietary intake ${ }^{(8,68)}$.

A narrative review that considered food pricing and affordability studies centred on whole diet costs conducted in New Zealand, Canada, the USA and the UK identified similar methodological concerns as described in our systematic review of the situation in Australia ${ }^{(8)}$.

There have been repeated calls for a nationally representative survey of the price and affordability of healthy foods in Australia since 2004, in order to highlight areas experiencing potential food insecurity due to high food prices $^{(6,9,12,67,69)}$. This will require the development of standardised tools and protocols that overcome many of the challenges identified in the current review ${ }^{(8,12)}$.

Such methods, aligning with the 'optimal' INFORMAS approach, are being finalised currently ${ }^{(69)}$. As an example of how these methods could be used to inform policy, they were used to investigate the impact of the potential extension of the $10 \%$ goods and services tax ${ }^{(70)}$ to basic healthy foods (that are currently exempt from the goods and services tax) in Australia. The study showed that the price differential between healthy (recommended) and current (less healthy) diets would increase markedly if this policy change was implemented as proposed ${ }^{(69,70)}$. Finalisation and application of these methods has the potential to overcome many of the identified problems of the previous 'healthy' food price assessment methods undertaken in Australia. 


\section{Limitations}

The present review was limited to those documents which were available from online searching. Additional food and diet pricing surveys may have been excluded if the reports were unavailable publicly. However, to our knowledge all of the major food pricing surveys, as well as many smaller, local surveys, have been located and analysed. Analysis of the food pricing survey was also limited to the reported information. In several instances details of data analysis or data collection methods were not reported.

\section{Conclusions}

Many 'healthy' food pricing surveys have been conducted in Australia. However, assessment methods vary across all metrics and most do not fully align with the recommendations of the current ADG. None have been applied nationally. Survey results are not comparable due to differences in the tools, protocols and methods and there is a need for a standardised national approach. Assessment of the price, price differential and affordability of a healthy diet (recommended) and current (unhealthy) diets would provide more robust and meaningful data to inform health and fiscal policy in Australia. The INFORMAS 'optimal' approach provides a potential framework for development of these methods.

\section{Acknowledgements}

Financial support: No financial support from any funding agency in the public, commercial or not-for-profit sector was provided for the systematic literature review. Financial support to ensure successful publication of the manuscript and to present findings at the national Healthy Diets ASAP stakeholder meeting in February 2016 was provided by The Australian Prevention Partnership Centre through the National Health Medical Research Council partnership centre grant scheme (grant number GNT9100001) with the Australian Government Department of Health, NSW Ministry of Health, ACT Health, HCF, and the HCF Research Foundation. The Australian Prevention Partnership Centre had no role in the design, analysis or writing of this manuscript. Conflict of interest: None. Authorship: Both authors jointly formulated the research question and designed the study. M.L. undertook the literature search and analysis and drafted the manuscript with supervision provided by A.L. Ethics of human subject participation: The QUT University Human Research Ethics Committee assessed this research as meeting the conditions for exemption from Human Research Ethics Committee review and approval in accordance with section 5.1.22 of the National Statement on Ethical Conduct in Human Research (2007; exemption number 1500000435).

\section{Supplementary material}

To view supplementary material for this article, please visit http://dx.doi.org/10.1017/S1368980016002160

\section{References}

1. Australian Institute of Health and Welfare (2015) Burden of overweight and obesity. http://www.aihw.gov.au/over weight-and-obesity/burden-of-disease/ (accessed October 2015).

2. National Health and Medical Research Council (2013) Eat for Health: Australian Dietary Guidelines. Canberra, ACT: Commonwealth of Australia.

3. Australian Bureau of Statistics (2014) 4364.0.55.007 Australian Health Survey: Nutrition First Results - Foods and Nutrients, 2011-12. http://www.abs.gov.au/AUSSTATS/ abs@.nsf/DetailsPage/4364.0.55.0072011- 12?OpenDocument (accessed June 2014).

4. Lee JH, Ralston RA \& Truby H (2011) Influence of food cost on diet quality and risk factors for chronic disease: a systematic review. Nutr Diet 68, 248-261.

5. Economic and Social Development Department, Food and Agriculture Organization of the United Nations (2003) Food security: concepts and measurement. In Trade Reforms and Food Security: Conceptualizing the Linkages, Chapter 2. http://www.fao.org/docrep/005/y4671e/y4671e06.htm\#fn31 (accessed October 2015).

6. Williams PG (2011) Can the poor in Australia afford healthy food? Nutr Diet 68, 6-7.

7. Burns C, Sacks G \& Gold L (2008) Longitudinal study of Consumer Price Index (CPI) trends in core and non-core foods in Australia. Aust N Z J Public Health 32, 450-453.

8. Lee A, Mhurchu CN, Sacks G et al. (2013) Monitoring the price and affordability of foods and diets globally. Obes Rev 14, 82-95.

9. Burns C \& Friel S (2007) It's time to determine the cost of a healthy diet in Australia. Aust N Z J Public Health 31, 363-365.

10. Hawkes C (2012) Food taxes: what type of evidence is available to inform policy development? Nutr Bull 37, 51-56.

11. Williams P, Reid M \& Shaw K (2004) The Illawarra Healthy Food Price Index: 1. Development of the food basket. Nutr Diet 61, 200-207.

12. Seal J (2004) Monitoring the price and availability of healthy food - time for a national approach. Nutr Diet 61, 197-198.

13. PRISMA (2015) Welcome to the Preferred Reporting Items for Systematic Reviews and Meta-Analyses (PRISMA) website! http://www.prisma-statement.org/ (accessed December 2015).

14. Ferguson M, O'Dea K, Chatfield M et al. (2015) The comparative cost of food and beverages at remote Indigenous communities, Northern Territory, Australia. Aust NZJ Public Health 40, Suppl. 1, S21-S26.

15. Leonard D, Zlotkowski K, Harrison M et al. (1997) The Healthy Food Access Basket Summary Report October 1997. Cairns, QLD: Queensland Health Tropical Public Health Unit.

16. Leonard D, Groos A \& Dunn S (1999) 1998 Healthy Food Access Basket Report. Cairns, QLD: Queensland Health Tropical Public Health Unit.

17. Lowry K (2003) Food Supply in South Western Sydney. Sydney, NSW: Sydney South West Area Health Service.

18. Queensland Government (2015) Healthy Food Access Basket Survey 2014, public release under development. Used with permission from Queensland Health. Brisbane, QLD: State of Queensland (Queensland Health). 
19. Friel S, Barosh LJ \& Lawrence M (2014) Towards healthy and sustainable food consumption: an Australian case study. Public Health Nutr 17, 1156-1166.

20. Queensland Health (2001) The Healthy Food Access Basket Survey 2000. Brisbane, QLD: Queensland Health.

21. McIntyre J (2006) Market Basket Survey Prepared for the Sydney Food Fairness Alliance (SFFA). Sydney, NSW: Centre for Population Health, Sydney West Area Health Service.

22. Palermo C \& Wilson A (2007) Development of a healthy food basket for Victoria. Aust N Z J Public Health 31, 360-363.

23. Meedeniya J, Smith A \& Carter P (2000) Food Supply in Rural South Australia: A Survey on Food Cost, Quality and Variety. Adelaide, SA: Eat Well SA - Food Supply in Rural South Australia.

24. Northern Territory Department of Health and Community Services, Nutrition and Physical Activity Program (2003) Market Basket Survey of Remote Community Stores in the Northern Territory April-June 2003. Darwin, NT: Department of Health and Community Services.

25. Cashel K \& Jeffreson S (1994) The Core Food Groups - The Scientific Basis for Developing Nutrition Education Tools. Canberra, ACT: National Health and Medical Research Council.

26. National Health and Medical Research Council (1992) Dietary Guidelines for Australians. Canberra, ACT: Commonwealth of Australia.

27. National Health and Medical Research Council (2011) $A$ Modelling System to Inform the Revision of the Australian Guide to Healthy Eating (FMS). Canberra, ACT: Commonwealth of Australia.

28. Pollard C \& Landrigan T (2011) Food Access and Cost Survey (FACS) Western Australia, 2010. Perth, WA: Department of Health, Western Australia.

29. Australian Bureau of Statistics (1998) National Nutrition Survey: Nutrient Intakes and Physical Measurements, Australia, 1995 (catalogue no. 4805.0). Canberra, ACT: Australian Bureau of Statistics.

30. Le Q, Murray S, Long G et al. (2013) Tasmanian Food Access Research Coalition (TFARC): Research Report. Hobart, TAS: Tasmanian Food Access Research Coalition.

31. Renzaho AMN (2008) Is a healthy diet affordable and accessible in the city of Yarra, Victoria-Australia? An analysis of cost disparity and nutritional choices. Ecol Food Nutr 47, 44-63.

32. Barosh L, Friel S, Engelhardt K et al. (2014) The cost of a healthy and sustainable diet - who can afford it? Aust $N Z J$ Public Health 38, 7-12.

33. Davidson A (2004) Katoomba Food Retail Price Mapping Project Report. Katoomba, NSW: Wentworth Area Health Service.

34. Kettings C, Sinclair AJ \& Voevodin M (2009) A healthy diet consistent with Australian health recommendations is too expensive for welfare-dependent families. Aust N ZJ Public Health 33, 566-572.

35. The Cancer Council NSW (2007) NSW Healthy Food Basket: Cost, Availability and Quality Survey. Sydney, NSW: Cancer Council NSW.

36. Chapman K, Kelly B, Bauman A et al. (2014) Trends in the cost of a healthy food basket and fruit and vegetable availability in New South Wales, Australia, between 2006 and 2009. Nutr Diet 71, 117-126.

37. Pollard C, Savage V, Landrigan T et al. (2015) Food Access and Cost Survey. Perth, WA: Department of Health, Western Australia.

38. Department of Human Services (2015) Online Estimators. http://www.humanservices.gov.au/customer/enablers/onlineestimators (accessed October 2015).

39. Marshall E, Morrison C \& Martin C (2010) Community Food Assessments in Zillmere. Brisbane, QLD: Queensland Health.

40. Sorbello C \& Martin C (2010) Bundaberg Community Food Assessment 2010. Brisbane, QLD: Queensland Health.
41. Australian Bureau of Statistics (2012) 6503.0 - Household Expenditure Survey and Survey of Income and Housing, User Guide, Australia, 2009-10. http://www.abs.gov.au/ ausstats/abs@.nsf/mf/6503.0/ (accessed July 2016).

42. Australian Bureau of Statistics (2005) 6530.0 - Household Expenditure Survey, Australia: Summary of Results, 2003-04. http://www.abs.gov.au/AUSSTATS/abs @.nsf/e8 ae5488b5988 39cca25682000131612/576c974dfba7243bca 25690b0081c163!OpenDocument (accessed October 2015).

43. Queensland Health \& Queensland Treasury (2007) The 2006 Healthy Food Access Basket (HFAB) Survey. Brisbane, QLD: Queensland Health.

44. Queensland Health \& Queensland Treasury (2012) The 2010 Healthy Food Access Basket (HFAB) Survey. Brisbane, QLD: Queensland Health.

45. Murray S, Ahuja K, Auckland S et al. (2014) The 2014 Tasmanian Healthy Food Access Basket (HFAB) Survey. Newnham, TAS: School of Health Sciences, University of Tasmania.

46. Australian Bureau of Statistics (2011) 2033.0.55.001 Census of Population and Housing: Socio-Economic Indexes for Areas (SEIFA), Australia, 2011. http://www. abs.gov.au/ausstats/abs@.nsf/Lookup/2033.0.55.001main+ features42011 (accessed April 2016)

47. Australian Population and Migration Research Centre (2015) ARIA and Accessibility. http://www.adelaide.edu.au/apmrc/ research/projects/category/aria.html (accessed April 2016).

48. Burns CM, Gibbon P, Boak R et al. (2004) Food cost and availability in a rural setting in Australia. Rural Remote Health 4, 311.

49. Wong KC, Coveney J, Ward P et al. (2011) Availability, affordability and quality of a healthy food basket in Adelaide, South Australia. Nutr Diet 68, 8-14.

50. Ward PR, Coveney J, Verity F et al. (2012) Cost and affordability of healthy food in rural South Australia. Rural Remote Health 12, 1938.

51. Tsang A, Ndung'u M, Coveney J et al. (2007) Adelaide Healthy Food Basket: a survey on food cost, availability and affordability in five local government areas in metropolitan Adelaide, South Australia. Nutr Diet 64, 241-247.

52. Palermo CE, Walker KZ, Hill P et al. (2008) The cost of healthy food in rural Victoria. Rural Remote Health 8, 1074.

53. Trezise J (2008) The Cost of Eating Well in the City of Greater Dandenong. https://www.accc.gov.au/system/files/ 026\%20-\%20Jenny\%20Trezise\%20\%2810\%20pages\%29.pdf (accessed September 2015).

54. Givoni N \& Palermo C (2010) Monitoring the cost of healthy food. Aust N Z J Public Health 34, 433-434.

55. Johnson C, Lewis E, McDonald G et al. (2008) Outer East Community Food Access Research Project: Food Security Assessment and Plans for a Way Forward. Melbourne, VIC: Monash University and Deakin University, C. Palermo and C. Smith.

56. De Rose K, Roberts S \& Nobes J (2011) Southern Grampians \& Glenelg Community Food Security Needs Assessment. Hamilton, VIC: Southern Grampians \& Glenelg Primary Care Partnership Food Security Working Group.

57. Lade R (2010) The Cost and Availability of Healthy Food in Wellington - May 2010. Wellington, VIC: Wellington Primary Care Partnership.

58. Pattieson D \& Palermo C (2010) Summary of 2010 Healthy Food Basket Data Across Various Local Government Areas in Victoria. Melbourne, VIC: Monash University.

59. Bradley V (n.d.) Access to Nutritious Food in the South Coast: Results of Victorian Healthy Food Basket Survey from 2010 to 2013. Wonthaggi, VIC: South Coast Primary Care Partnership.

60. Thomson D (2013) Healthy Together Bendigo Food Security Report. Bendigo, VIC: Healthy Food Connect.

61. Stirrat A (2012) Food Security - Building the Local Picture A Needs Assessment Report. Geelong: VIC: City of Greater Geelong. 
62. Northern Territory Department of Health and Community Services, Nutrition and Physical Activity Program (2014) Market Basket Survey of Remote Community Stores in the Northern Territory April 2014. Darwin, NT: Department of Health and Community Services.

63. Northern Territory Department of Health and Community Services, Nutrition and Physical Activity Program (2012) Market Basket Survey of Remote Community Stores in the Northern Territory April-June 2012. Darwin, NT: Department of Health and Community Services.

64. Williams P, James Y \& Kwan J (2004) The Illawarra Healthy Food Price Index 2. Pricing methods and index trends from 2000-2003. Nutr Diet 61, 208-214.

65. Williams P, Hull A \& Kontos M (2009) Trends in affordability of the Illawarra Healthy Food Basket 2000-2007. Nutr Diet 66, 27-32.

66. Queensland Health (2002) The 2001 Healthy Food Access Basket (HFAB) Survey. Brisbane, QLD: Queensland Health.

67. Palermo C (2011) The cost of nutritious food: a determinant of health. Nutr Diet 68, 246-247.

68. Australian Bureau of Statistics (2014) Australian Health Survey: Nutrition First Results - Foods and Nutrients, 2011-12. Foods Consumed. http://www.abs.gov.au/ ausstats/abs@.nsf/Lookup/4364.0.55.007main+features2201112, accessed February 2016.

69. Lee A, Kane S, Ramsey R et al. (2016) Testing the price and affordability of healthy and current (unhealthy) diets and the potential impacts of policy change in Australia. BMC Public Health 16, 315.

70. Australian Government, The Treasury (2015) Re:think Tax Discussion Paper. Canberra, ACT: Commonwealth of Australia, Department of Treasury.

71. Queensland Health (2005) The 2004 Healthy Food Access Basket (HFAB) Survey. Brisbane, QLD: Queensland Health.

72. Queensland Health \& Queensland Treasury (2012) The 2010 Healthy Food Access Basket (HFAB) Survey. J Home Econ Inst Aust 19, 12-24.

73. Lee AJ, Darcy AM, Leonard D et al. (2002) Food availability, cost disparity and improvement in relation to accessibility and remoteness in Queensland. Aust NZ J Public Health 26, 266-272.

74. Harrison M, Coyne T, Lee A et al. (2007) The increasing cost of the basic foods required to promote health in Queensland. Med J Aust 186, 9-14.

75. Harrison M, Lee A, Findlay M et al. (2010) The increasing cost of healthy food. Aust $N Z J$ Public Health 34 , 179-186.

76. Pollard CM, Landrigan TJ, Ellies PL et al. (2014) Geographic factors as determinants of food security: a Western Australian food pricing and quality study. Asia Pac J Clin Nutr 23, 703-713.

77. Northern Territory Department of Health and Community Services, Nutrition and Physical Activity Program (2004) Market Basket Survey of Remote Community Stores in the Northern Territory April-June 2004. Darwin, NT: Department of Health and Community Services.

78. Northern Territory Department of Health and Community Services, Nutrition and Physical Activity Program (2005) Market Basket Survey of Remote Community Stores in the Northern Territory April-June 2005. Darwin, NT: Department of Health and Community Services.
79. Northern Territory Department of Health and Community Services, Nutrition and Physical Activity Program (2006) Market Basket Survey of Remote Community Stores in the Northern Territory April-June 2006. Darwin, NT: Department of Health and Community Services.

80. Northern Territory Department of Health and Community Services, Nutrition and Physical Activity Program (2007) Market Basket Survey of Remote Community Stores in the Northern Territory April-June 2007. Darwin, NT: Department of Health and Community Services.

81. Northern Territory Department of Health and Community Services, Nutrition and Physical Activity Program (2008) Market Basket Survey of Remote Community Stores in the Northern Territory April-June 2008. Darwin, NT: Department of Health and Community Services.

82. Northern Territory Department of Health and Community Services, Nutrition and Physical Activity Program (2009) Market Basket Survey of Remote Community Stores in the Northern Territory April-June 2009. Darwin, NT: Department of Health and Community Services.

83. Northern Territory Department of Health and Community Services, Nutrition and Physical Activity Program (2010) Market Basket Survey of Remote Community Stores in the Northern Territory April-June 2010. Darwin, NT: Department of Health and Community Services.

84. Northern Territory Department of Health and Community Services, Nutrition and Physical Activity Program (2011) Market Basket Survey of Remote Community Stores in the Northern Territory April-June 2011. Darwin, NT: Department of Health and Community Services.

85. McLean E, Annat K \& Lee A (2014) Store Nutrition Report and Market Basket Survey April 2014. Ciccone, NT: Nganampa Health Council.

86. Lee A, Rainow S, Tregenza J et al. (2016) Nutrition in remote Aboriginal communities: lessons from Mai Wiru and the Anangu Pitjantjatjara Yankunytjatjara Lands. Aust $N Z J$ Public Health 40, Suppl. 1, S81-S88.

87. Williams P (2010) Monitoring the affordability of healthy eating: a case study of 10 years of the Illawarra Healthy Food Basket. Nutrients 2, 1132-1140.

88. Palermo C, Perera-Schulz D, Kannan A et al. (2014) Development and evaluation of an iPad app for measuring the cost of a nutritious diet. JMIR Mhealth Uhealth 2, e50.

89. Ward PR, Verity F, Carter P et al. (2013) Food stress in Adelaide: the relationship between low income and the affordability of healthy food. J Environ Public Health $\mathbf{2 0 1 3}$, 968078.

90. Angarane T \& Bradley V (2010) The Cost and Availability of Food in Bass Coast and South Gippsland, Victoria. Wonthaggi, VIC: South Coast Primary Care Partnership.

91. McCloy N \& Bradley V (2010) The Cost and Availability of Food in Bass Coast and South Gippsland, Victoria. Wonthaggi, VIC: South Coast Primary Care Partnership.

92. Bradley V (2010) Victorian Health Food Basket Survey in the Bass Coast and South Gippsland 2008 and 2009. Wonthaggi, VIC: South Coast Primary Care Partnership.

93. Chambers M \& Fedele W (2014) Food Security in Port Melbourne A report to investigate access to bealthy food. Melbourne, VIC: La Trobe University \& Inner South Community Health. 